\title{
Chants individuels et chants collectifs chez les Kaiapô du Brésil central
}

\author{
Gustaaf Verswijver \\ Traducteur : Isabelle Schulte-Tenckhoff
}

\section{OpenEdition \\ Journals}

Édition électronique

URL : http://journals.openedition.org/ethnomusicologie/2293

ISSN : 2235-7688

Éditeur

ADEM - Ateliers d'ethnomusicologie

Édition imprimée

Date de publication : 1 janvier 1988

Pagination : 13-27

ISBN : 2-8257-0159-9

ISSN : $1662-372 X$

Référence électronique

Gustaaf Verswijver, "Chants individuels et chants collectifs chez les Kaiapô du Brésil central », Cahiers d'ethnomusicologie [En ligne], 1 | 1988, mis en ligne le 15 août 2011, consulté le 01 mai 2019. URL : http://journals.openedition.org/ethnomusicologie/2293 


\title{
CHANTS INDIVIDUELS ET CHANTS COLLECTIFS CHEZ LES KAIAPÓ DU BRÉSIL CENTRAL*
}

\author{
Gustaaf Verswijver
}

Il y a actuellement plus de trois milles Indiens Kaiapó établis dans treize villages. Les Kaiapó appartiennent à la branche septentrionale du groupe linguistique Gê. Traditionnellement, les Gê du nord occupaient une vaste région au Brésil central, et ils partagent de nombreux traits sociaux et linguistiques.

Les Kaiapó connaissent une panoplie de chants, mais peu d'anthropologues se sont intéressés jusqu'ici à leur musique, voire même à celle des Gê en général ${ }^{1}$. Sans être moi-même ethnomusicologue, je me propose ici de donner un aperçu de la musique vocale des Kaiapó, centré sur une typologie des chants, y compris leurs modes de transmission. Parallèlement, j'évoquerai la manière dont émergent de nouveaux chants. Enfin je donnerai, chaque fois que ceci est possible, la transcription littéraire d'un chant illustrant chaque type retenu. L'ensemble de ces observations est basé sur mes enquêtes de terrain menées chez les Kaiapó entre 1974 et $1981^{2}$.

Sans être exhaustive, la série de chants kaiapó présentée dans ces pages comprend la majorité des chants normalement interprétés. Pour mieux comprendre certains points soulevés en cours de démonstration, il est utile de passer brièvement en revue les principaux aspects de la vie rituelle des Kaiapó.

\section{A propos des rites kaiapó}

Traditionnellement, les villages kaiapó consistent en un cercle de maisons se dressant à intervalles réguliers autour d'une grande place centrale. Le centre du village kaiapó est marqué par la maison des hommes où les associations masculines se réunissent tous les jours ${ }^{3}$.

Le village représente le centre de l'univers kaiapó : c'est l'espace socialisé par excellence. La forêt alentour est considérée comme un univers non social peuplé d'êtres mi-hommes mi-animaux, où les humains risquent de se muer en animaux ou en esprits, de devenir fous et de tuer un membre de leur parenté. Plus on s'éloigne du village, plus ces dangers sont une réalité et plus la forêt devient non sociale. D'où la nécessité de socialiser cette partie de la forêt 
que les villageois fréquentent régulièrement pour se déplacer, se rendre aux jardins, chasser ou pêcher. Cette socialisation est accomplie en nommant certains lieux ${ }^{4}$ et en conduisant une série de rites soit directement en forêt, soit avec l'objectif de socialiser un type particulier d'intervention sur celle-ci.

Les Kaiapó pratiquent de nombreux rites dont la portée et la durée peuvent cependant varier considérablement. En plus d'une série de rites de passage solennels et souvent fort brefs - laissés de côté ici - qui visent à marquer et à reconnaître publiquement le passage d'une classe d'âge à une autre $^{5}$, les rites les plus importants portent sur l'articulation de catégories sociales, ou encore sur la socialisation des interventions sur la forêt non sociale.

Avant d'atteindre l'adolescence, garçons et filles peuvent être «honorés » par une série de cérémonies importantes. Le terme kaiapó correspondant à ce que je traduis ici par "honorés" est me rer mex (littéralement "ceux qui font étalage de leur beauté "); il se réfère à la manière souvent fort élaborée dont ces jeunes se parent durant l'étape finale de la cérémonie. Les enfants «honorés» sont le point de mire des cérémonies d'imposition de noms ainsi que de la pêche ritualisée.

Les Kaiapó distinguent deux catégories de noms individuels : idji kakrit ( "noms communs») et idji mex ("noms beaux»). Quelques jours après la naissance, certains membres du groupe de parenté confèrent au nouveau-né une série de noms tant «communs » que «beaux». Cette dernière catégorie exige par la suite d'être confirmée dans le cadre d'une cérémonie d'imposition de noms, organisée par les parents des enfants "honorés». Idéalement, un enfant sera honoré au cours de diverses cérémonies et, à nouveau, au moment de son initiation.

Le rituel initiatique implique simultanément une initiation, un mariage rituel et, parfois, un rite de passage d'une classe d'âge à une autre. L'initiation n'est jamais organisée isolément ; elle est plutôt intégrée à l'une des deux principales cérémonies d'imposition de noms, ou encore à la cérémonie élaborée que représente la pêche rituelle marquant la fin de la saison sèche.

En règle générale, deux ou trois cérémonies d'imposition de noms sont organisées chaque année. Le nombre d'enfants honorés à ces occasions varie entre un et onze, la moyenne se situant entre deux et cinq.

Les cérémonies d'imposition de noms ainsi que la pêche rituelle se caractérisent toutes deux par le fait que plusieurs individus passent d'une catégorie

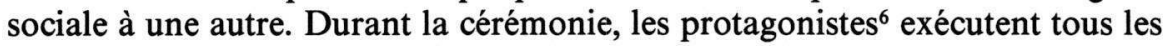
jours des danses brèves ou se réunissent pour chanter ensemble. L'étape finale de ces cérémonies est marquée par une grande danse qui dure généralement toute la nuit. Pour cette occasion, tous les danseurs se parent d'une variété d'ornements impressionnants'.

La cérémonie annuelle du maïs est organisée pour stimuler la maturation du maïs, et elle peut s'étaler sur trois mois. Par contre, les cérémonies liées aux autres types d'interventions dans la nature (par exemple, défrichement de nouveaux terrains de jardinage ou chasse au jaguar) durent rarement plus longtemps qu'une ou deux journées. 


\section{La musique vocale des Kaiapó}

C'est par le terme de ngre-re que les Kaiapó désignent la musique tant vocale qu'instrumentale. Dans le présent essai, je me borne toutefois à la première ${ }^{8}$.

Les Kaiapó n'ont pas coutume de chanter pour le seul plaisir: par exemple, on voit rarement un individu exprimer son bonheur par le chant. $\mathrm{Ce}$ dernier appartient en effet aux occasions formelles (cérémonies, discours, apprentissage que traverse un garçon ou un jeune homme, etc.).

La musique vocale kaiapó peut être subdivisée en deux catégories, soit les chants que l'on pourrait qualifier de restreints parce que réservés à certains individus, et les chants communautaires que tout un chacun est autorisé à interpréter. L'une et l'autre comportent en outre un certain nombre de souscatégories déterminées par les circonstances dans lesquelles les chants sont interprétés, ainsi que par leur mode de transmission.

\section{Les chants « restreints»}

Les chants dont la connaissance aussi bien que l'interprétation sont réservées à un petit nombre d'individus, possèdent tous un modèle fixe. D'autre part, ils ne sont jamais interprétés en falsetto, et leur transmission s'accomplit invariablement à l'intérieur du cercle tracé par les maisons.

Pour mieux voir les différences entre les types de ces chants «restreints", il convient de se représenter tout d'abord les catégories de personnes qui sont autorisées à les interpréter en public.

Les chefs des associations masculines. Chaque association se dote d'un chef au moins, mais nombre d'entre elles sont dirigées par deux chefs dont chacun s'entoure alors d'une suite. Ces chefs (benjadjwỳr), qui remplissent des fonctions rituelles et politico-juridiques, sont appelés à illustrer l'idéal masculin kaiapó, fondé sur des vertus comme la sagesse, l'éloquence, l'intrépidité, la solidarité et la générosité.

Les Kaiapó n'observent nulle règle formelle quant aux modalités de la succession à la chefferie, mais ils accordent la préférence à la transmission de cette fonction au fils ou au neveu (biologique ou classificatoire) du chef. Ainsi les chefs œuvrent-ils patiemment pour enseigner à certains de ces jeunes le vaste répertoire des connaissances spécialisées que leur fonction exige. Un tel apprentissage dure généralement des années. Quant à la question de savoir lequel parmi les jeunes gens formés de cette manière exercera la chefferie, la décision obéit à un long processus; elle dépend notamment de facteurs tels que l'ambition personnelle du jeune homme et le soutien dont il bénéficie chez ses pairs au sein de l'association masculine (soutien qu'il peut s'assurer en adoptant un comportement exemplaire).

Les hommes dotés de privilèges rituels. Une série d'hommes détiennent des privilèges rituels entraînant le droit d'interpréter certains chants dans un cadre cérémoniel. La transmission de ces prérogatives se limite à des catégories spécifiques de parenté 9 .

Les maîtres du rituel. Parmi tous les privilèges rituels, la fonction de maître du rituel, dont l'équivalent kaiapó est ngrenhõdjwỳnh ou «vrai chanteur», est 


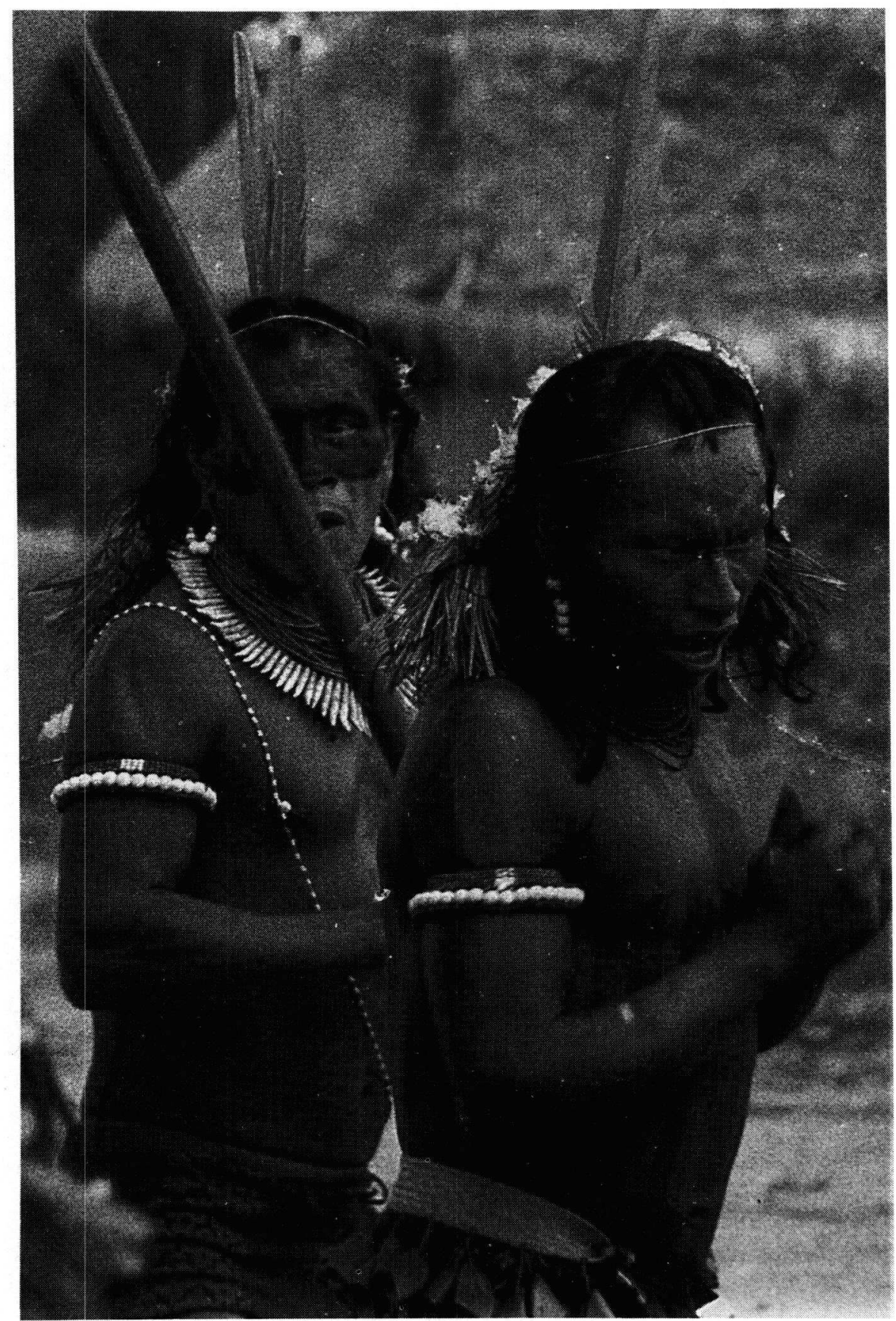

Deux maîtres du rituel menant la danse oportirekô en jouant du hochet. Kaiapó-Mekrãgnotí. P.I. Mekranoti, Brésil central, 1978 (photo : Gustaaf Verswijver). 
celle qui véhicule le plus grand prestige. Les maîtres du rituel connaissent un grand nombre de chants, et on leur demande souvent conseil à ce sujet. Dépositaires d'une grande partie du savoir relatif aux danses et aux chants, ils sont indispensables à la plupart des cérémonies importantes. Ce sont eux qui mènent généralement la danse et qui tiennent le hochet ${ }^{10}$ à l'occasion d'une série de cérémonies publiques.

Chaque communauté kaiapó a souvent plusieurs maîtres du rituel qui, durant les grandes cérémonies, s'acquittent conjointement de leurs devoirs. Chaque maître du rituel choisit parmi la catégorie des petits-fils ou fils de la sœur un ou plusieurs garçons qu'il souhaite former. L'apprentissage commence lorsque l'enfant est encore très jeune, et il s'étale sur plusieurs années. Un maître du rituel récemment formé ne deviendra cependant actif qu'à l'âge adulte, c'est-à-dire après avoir acquis une base solide d'expériences et de prestige.

On peut distinguer trois types de chants "restreints" en fonction de l'occasion à laquelle ils sont interprétés, ainsi que de leur contenu et de leur forme, types que je désignerai arbitrairement comme "chants rituels", «chants de purification » et «chants d'honneur».

Le premier type renvoie à ce que les Kaiapó appellent ben, terme qui se réfère à une série de formules stylisées chargées de pouvoirs spéciaux. L'interprétation des ben constitue l'une des principales fonctions rituelles du chef, comme l'illustre le terme kaiapó désignant la chefferie, soit benjadjwỳr (littéralement, "celui qui chante vraiment le ben») ou ir-re (abréviation de bendjír, «celui qui place le iben»). Ce type de chant peut être interprété à diverses occasions et prendre des formes variées, mais seule une catégorie nous intéresse ici, soit les adresses rythmées, semblables à des récitatifs qui, du point de vue de leur contenu, comprennent un ou plusieurs des éléments suivants : rémontrances, incitation à se préparer pour les danses, à exécuter celles-ci d'une manière appropriée, à arborer les parures requises, etc., auxquelles il faut ajouter les formules rituelles destinées à prévenir une calamité annoncée par un phénomène naturel.

Les Kaiapó croient, en effet, que le fait d'entendre ou de voir un météore s'écraser sur le sol, ou encore de voir deux lignes rouges apparaître dans le ciel, annoncent une calamité imminente. Tous les hommes se rassemblent alors pour exécuter une brève danse, tandis que le chef - qui se tient à l'extérieur du cercle des danseurs - interprète un ben dont on croit qu'il conjure le malheur.

Pendant certaines cérémonies d'imposition de noms, les ben sont interprétés par un seul chef qui est soit le chef de l'association masculine la plus éminente, soit un parent de l'enfant destiné à recevoir un nom. Assis dans la maison des organisateurs de la cérémonie, le chef fait face à l'enfant qui est tenu par sa mère. Pareil chant rituel peut durer dix minutes ou plus, et il est répété pour chacun des enfants auxquels s'adresse la cérémonie. Si toutes les cérémonies ne s'accompagnent pas nécessairement de chants rituels, ceux-ci, lorsqu'ils sont interprétés, sont considérés comme étant aussi importants que l'imposition de noms elle-même. 
Voyons maintenant l'extrait d'un ben de douze minutes, interprété par un chef éminent à l'occasion d'une cérémonie d'imposition de noms enregistrée en 1976. Cet extrait montre clairement deux éléments propres à ce type de chant rituel : les rémontrances et les exhortations.

Go ga ar ã̃ ikàtxi tã abê bikukwa-jê kurê towa nhyr karõ-ri ba.

Ba ibê xakrãtũmmũ-re.

Ajben ne tã ajakre to nhy.

Amu amĩ tãj mar bê ba nhô mry my no ngrekà ja kãm mã.

Ba i-ben ne kãm-mã xamĩ rĩt-tĩ mã ri ba.

Ri ba amĩ to djà kukajêr kãm mã'ã i-ben ne to amĩ pyràk.

Gê abêkukwa ja gê kute amĩ kraxi kêtê.

Gê kute amĩ rotã.

Gê amĩ to mõ amĩ to bôx.

Nễ amĩ tãjbê xakrãtũmmü-re ben nẽ mã.

Gê kute ba nhõ mry kàpũtĩ.

Kôt tô bixi amak kỳ ben nẽ.

Gê kute ba nhõ ngykajkriti kôt bixi amak kỳ ben nẽ.

Gê amĩ tomõ nẽ amĩ tãjbê xakrãtũmmũ-re ben nẽ ma.

Ne vous battez pas devant moi avec vos parents pour les privilèges rituels. Je suis le grand-père de vous tous.

Je vous chante le ben.

Vous savez tous comment il faut danser et de quels ornements il faut se parer pour être beau.

Je ne cache pas mon ben, je le récite au milieu de vous tous.

J'ai voulu apprendre le ben,

Et j'ai demandé à notre grand-père (de me le dire) chaque jour.

Si votre parent pauvre vous demande un privilège rituel, alors donnez-le lui.

Donnez quelque-chose aux pauvres.

Faites approcher les pauvres.

Faites-leur écouter mon ben.

Ces pauvres qui chassent seulement des animaux ${ }^{11}$.

Ils ne pensent qu'à cela.

Ils ne pensent qu'à manger du miel.

Faites-les venir, je suis leur grand-père.

$\mathrm{Si}$, au cours d'une cérémonie d'imposition de noms, les chefs des associations masculines n'interprètent pas leurs ben, le maître du rituel exécute un autre type de chant rituel. Se tenant debout sur la place du village, un hochet ou un bâton muni de sonnailles à la main, il chante quelques vers courts. $\mathrm{Ce}$ chant est répété pour chacun des enfants auxquels s'adresse la cérémonie.

Quant au deuxième type de chants «restreints» considérés ici, il arrive parfois qu'on sollicite un chef d'interpréter un "chant de purification", notamment lorsqu'on ramène au village le butin d'un raid, que l'on reçoit des 
dons de la part de parents établis dans d'autres villages kaiapó, ou encore après avoir confectionné un ornement important fait de plumes. Pour chaque catégorie d'objets il existe un chant spécifique de purification, et les Kaiapó disent qu'à défaut d'exécuter le chant requis, l'utilisation de l'objet en question mettrait en péril la vie de son propriétaire ou de ses parents proches. Ainsi les chants de purification sont-ils interprétés, non seulement pour reconnaître l'acquisition d'un objet étranger, mais encore pour le socialiser.

Les chants en question sont toujours exécutés devant l'objet. Ils sont plutôt brefs, comme l'atteste l'exemple suivant d'un chant de purification destiné à socialiser une couronne de plumes jaunes (pêjàtijamy) nouvellement confectionnée :

To nhym me na àkkà bikàr-re.

To nhym me na àkkà bikàr-re.

Pêjàtijamy'ã djàkkà mexti pyràk.

To nhym me na àkkà bikàr-re.

To nhym me na àkkà bikàr-re.

Qui a fabriqué cette couronne?

Qui a fabriqué cette couronne?

Elle est belle à voir avec ses plumes jaunes.

Qui a fabriqué cette couronne?

Qui a fabriqué cette couronne?

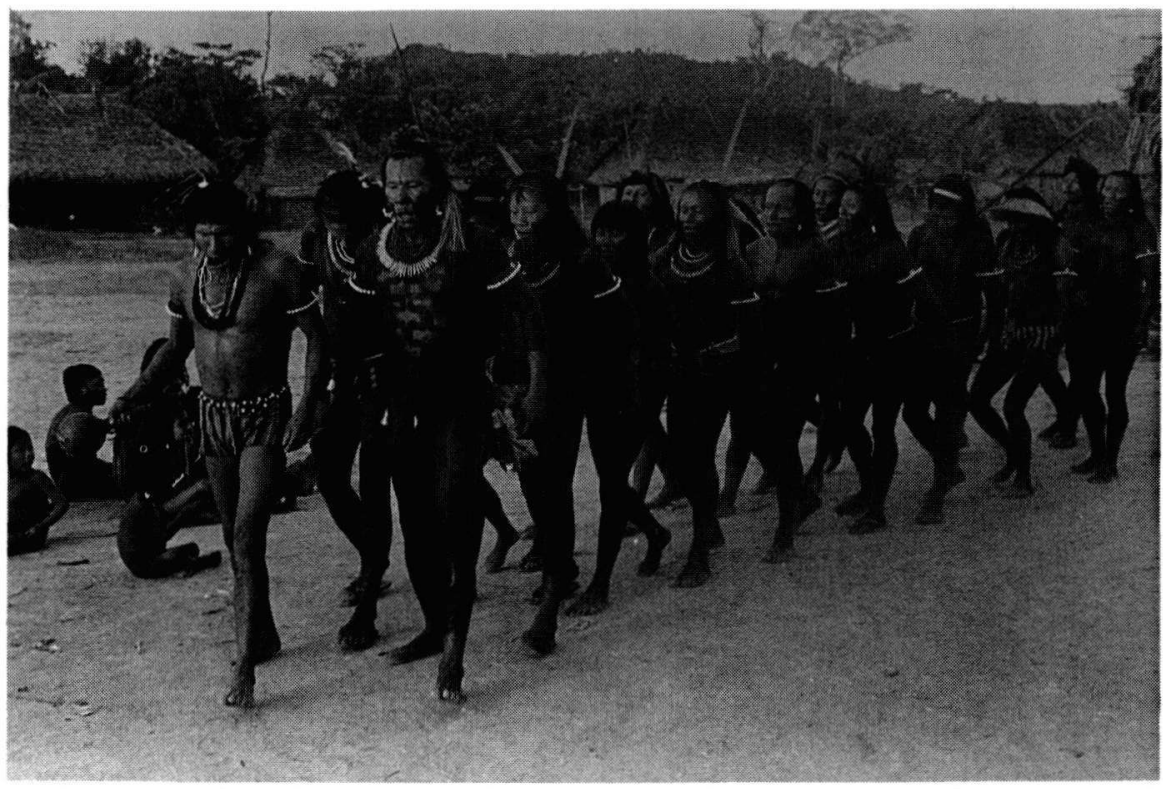

Tàkàkma-re chantant lors de la cérémonie du maïs bàyjangri. Kaiapó-Mekrãgnotí. P.I. Mekranoti, Brésil central, 1979 (photo : Gustaaf Verswijver). 
Il est extrêmement rare aujourd'hui de voir un chef interpréter un chant de purification, sans doute en raison des quantités toujours plus importantes de biens de consommation introduites chez les Kaiapós depuis leur «pacification".

Alors que les chefs font porter leurs chants de purification sur les objets, les maîtres du rituel les adressent à la nourriture. C'est ainsi qu'ils exécutent un large éventail de chants de purification au moment de la première récolte. Une femme de chaque famille nucléaire apporte quelques produits du jardin au maître du rituel qui, le hochet à la main, chante quelques vers afin de bénir les plantes, c'est-à-dire d'exorciser leurs « esprits " ${ }^{12}$ susceptibles de provoquer des maladies. La famille nucléaire en question est ensuite autorisée à faire la récolte et à consommer le produit sans devoir craindre la maladie.

Les chants d'honneur, enfin, illustrent le privilège rituel détenu par certains hommes de chanter «en l'honneur de l'enfant». Ce chant, qui ne dure le plus souvent que quelques minutes, est interprété soit sur la place du village, soit dans la maison où habitent les enfants auxquels est destinée la cérémonie ; dans ce dernier cas, le chanteur, son hochet à la main, exécute une série de pas devant les enfants.

\section{Les chants communautaires}

Les chants communautaires, que tout un chacun peut interpréter en public (les seules restrictions à ce sujet étant liées au sexe de l'interprète), sont exécutés individuellement ou en groupe.

Sur la base des différentes occasions auxquelles ces chants sont réservés, je propose de distinguer: les chants cérémoniels, les chants d'annonce et les chants anciens.

Pour ce qui est des chants cérémoniels, il convient de préciser tout d'abord que chaque cérémonie kaiapó est assortie d'une série de chants qui lui est exclusive $^{13}$. Il faut cependant établir une différence entre les chants communautaires à modèle fixe et ceux dont les paroles peuvent varier. Pour mieux saisir ces différences, il n'y a pas meilleur exemple que les divers chants communautaires exécutés durant la cérémonie d'imposition de noms appelée tàkàk/nhàk ${ }^{14}$.

La plupart des chants communautaires kaiapó ont un modèle fixe, c'est-àdire que la mélodie est préétablie, de même que la disposition des vers qui sont, eux aussi, invariables. L'idéal kaiapó consiste à considérer ces chants comme étant immuables dans le temps; mais cette immutabilité n'en est pas moins relative.

Lors des préparatifs en vue de toute cérémonie importante, il y a généralement débat dans la maison des hommes. Les aînés et les maîtres du rituel discutent des divers aspects de la cérémonie à venir : son moment propice, la manière de conduire les danses et les rites, les ornements qu'il faut porter, et ainsi de suite. Mais souvent, le point essentiel de tout l'argument concerne la question de savoir quels chants interpréter. 
D'une manière générale, les grandes cérémonies kaiapó s'accompagnent d'une série de chants de durée variable : «brefs chants communautaires » qui ne comptent que quelques vers et font rarement l'objet de la discussion, et "grands chants communautaire» pouvant durer jusqu'à vingt minutes. Le plus souvent, c'est en fait la séquence «appropriée» des vers des grands chants qui est sujette à controverses et que l'on débat sur un ton animé à l'abri de la maison des hommes.

Pareilles discussions sont plus agitées encore lorsqu'il s'agit de préparer une cérémonie qui n'a pas eu lieu depuis longtemps ${ }^{15}$, au sujet de laquelle les points de vue des aînés ou des maîtres du rituel sont susceptibles de diverger considérablement en ce qui concerne l'enchaînement des vers. D'une manière similaire, il n'est pas rare de voir certains vers tomber dans l'oubli et être graduellement remplacés par de nouveaux ${ }^{16}$.

Une fois parvenu à un compromis sur la séquence des vers, les Kaiapó se mettent à répéter les grands chants communautaires pendant des semaines d'affilée, en organisant des sessions journalières de chant collectif. Cette longue période de répétition permet aux jeunes d'apprendre les chants; et elle est toujours une affaire publique puisqu'elle se déroule sur la place du village, dans la maison des hommes ou dans les campements en forêt au moment des randonnées. Au cours de la répétition, les chants sont généralement exécutés sur le registre grave, alors que leur interprétation cérémonielle se fait sur le registre aigu, voire même en falsetto, tout en étant accompagnée de danses à participation nombreuse.

Les exemples qui suivent illustrent les «brefs chants communautaires " à modèle fixe. Ils furent enregistrés en 1975 au moment de la cérémonie d'imposition de noms tàkàk/nhàk. Durant cette partie de la cérémonie, un homme figurant un jaguar est présent :

Ha'ê'êe.

Ha'ê'êê.

Djana ga me rob mã àkkà pumu.

Djana ga me rob mã àkkà pumu.

Kên-ne ngâ krô nhĩja rummu.

Nã rob o pumati-re àkkà prãmmã tê mõ nẽ bôx.

Ikatxêt-te he.

Ikatxêt-te he.

Djana ga me amĩ mã prĩ.

Ba rôrekre-re kammã krwỹnhkakti-re

ngwỳnhnhĩ kwỳ by.

Ga ja me to ge katàmmà katxàkkà.

Ikatxêt-te.

Ha'ê'ê'ê.

As-tu vu le jaguar avec sa couronne de plumes.

Venant de l'endroit rempli de pierres.

Le jaguar peureux est allé chercher la couronne qui lui plaît. 
Tout peint que tu sois,

Après avoir marché lentement.

Je prendrai le duvet du perroquet dans le trou des termites.

Pour que tu t'en pares pour la danse et ne ressembles pas à un singe.

Tout peint que tu sois.

D'autres cérémonies, en revanche, sont assorties de chants qui changent à chaque fois. Durant la cérémonie d'imposition de noms tàkàk/nhàk, des enfants - dont le nombre varie entre un et six - voient leurs "noms beaux" confirmés. Comme il a été signalé plus haut, les noms à confirmer durant une cérémonie d'imposition de noms sont ceux attribués à la naissance. Néanmoins, il arrive souvent qu'un certain nombre de nouveaux noms émergent pendant les préparatifs en vue de la cérémonie en question. Ce sont les chamanes qui les apportent à la communauté.

On dit que les chamanes sont capables de prendre contact avec les esprits humains et animaux ainsi qu'avec d'autres éléments de la nature (telles la pluie ou les feuilles de certains arbres), de les voir et de les consulter. En communiquant avec ces esprits, les chamanes apprennent de nouveaux remèdes, s'enquièrent de la localisation d'ennemis et de leur identité, etc.

Un individu devient chamane à la suite d'une expérience personnelle telle une maladie grave - parce qu'on croit que son esprit a quitté son corps pour le réintégrer ensuite. Le savoir chamanique, qui consiste à jeter des sorts, voire à neutraliser ceux-ci, ainsi qu'à susciter le retour d'un esprit ayant quitté un malade, équivaut à un pouvoir exceptionnel qui place le chamane à l'écart des autres membres de la société.

L'un des principaux "grands chants communautaires» de la cérémo-

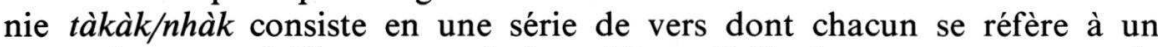
«nom beau» spécifique assorti du préfixe tàkàk. Au commencement de la cérémonie, les vers correspondent à ceux chantés à l'occasion de la cérémonie précédente du même type. Mais au cours de la première semaine, de nouveaux vers s'y substituent graduellement, qui ont été apportés à la communauté par des chamanes les ayant appris en fréquentant l'esprit d'un animal ou d'un élément de la nature doté d'un nom muni du préfixe tàkàk.

Le plus souvent, les chamanes communiquent avec ces esprits en faisant un rêve ou en allant dans la forêt. Ils parlent aux esprits qui leur apprennent ensuite leur nom. Ces noms nouvellement introduits dans la communauté cérémonielle sont toujours mentionnés dans les vers qui révèlent aussi l'identité de l'animal ou de l'élément de la nature dont ils proviennent.

Les nouveaux vers sont interprétés par le chamane à plusieurs reprises dans la maison des hommes, pour être ensuite incorporés au chant communautaire et rendus publics le même jour au moment de la danse.

Un chamane peut introduire plusieurs nouveaux noms (et leurs vers correspondants), mais ceux-ci ne sont pas d'emblée ceux qui seront confirmés durant la cérémonie en cours, étant donné que les noms sont invariablement 


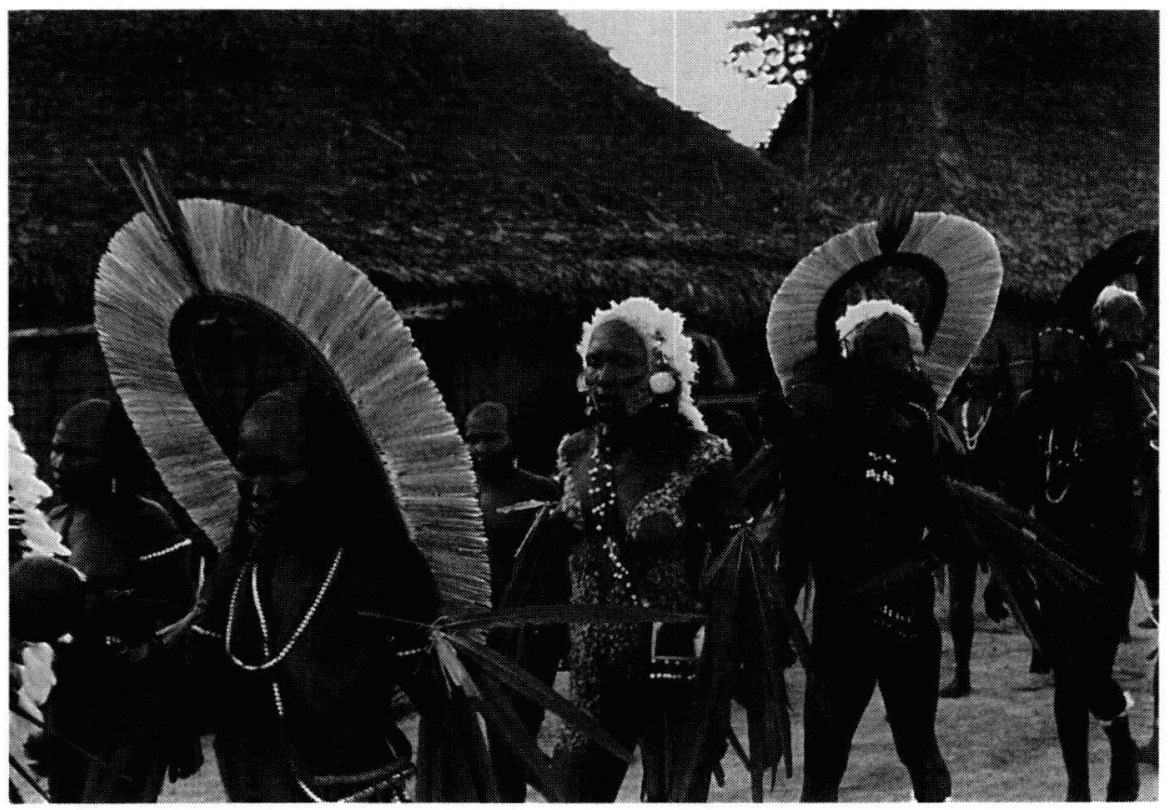

Les femmes lors de la phase finale de la cérémonie d'imposition de noms me ni bi'ôk. Kaiapó-Mekrãgnotí. P.I. Mekranoti, Brésil central, 1976 (photo : Gustaaf Verswijver).

transmis d'un homme à ses petits-enfants ou neveux; les chamanes qui apportent de nouveaux noms peuvent transmettre et confirmer ceux-ci à condition que leurs petits-enfants ou neveux soient «honorés " durant la cérémonie en cours. Si tel n'est pas le cas, la transmission des noms se fait sous réserve de leur confirmation rituelle à l'occasion d'une cérémonie tàkàk/nhàk ultérieure.

Durant la cérémonie tàkàk/nhàk qui se déroula en 1975, par exemple, huit nouveaux noms furent introduits par les chamanes, mais trois seulement furent transmis aux enfants auxquels s'adressait la cérémonie. Voici quelques vers enregistrés à cette occasion.

\section{Nĩangdjyti-re nhĩnhĩ bê}

Tàkàkànõrti-re jã.

Aringrô tỳx,

Jakamàn nã Tàkàkkà-re ja mỳr õtõnõ.

Dja me ba kêtte-ri

nã mry kamrêkti-re kute me ba kàjmãtã djwỳ-re bôr mã katôr.

Nhynhiryti-re nhĩnhĩ bê nã Tàkàkkàkajkritti-re nã ba me ngipôkuri kutxêt nẽ. 
Le nom du grand cerf rouge est Tàkàkànõrti-re.

Dans la chaleur de la journée

il (le cerf-tàkàk) n'arrête pas de pleurer.

\begin{abstract}
Avant notre temps
nos ancêtres mangeaient leur viande crue, car ils ne savaient pas la cuire.

Le nom du vautour était Tàkàkkàkajkritti-re

et il n'arrêtait pas de dessiner des cercles au-dessus des hommes.
\end{abstract}

Le deuxième type de chants communautaires est constitué des chants d'annonce. Par exemple, lorsqu'un chasseur a abattu du gibier, il entonne un chant sur le chemin de retour au village ou au campement pour annoncer le succès de son entreprise. Pour chaque animal chassé il existe un type particulier de chant.

Les chants d'annonce véhiculent toujours un message et sont aussi interprétés lorsqu'un groupe de guerriers retourne d'un raid, qu'un groupe d'hommes revient d'une longue randonnée en forêt, ou qu'un homme (ou un groupe d'hommes) désire informer les villageois d'un événement spécial, tel un danger décélé dans les environs du village ou le fait qu'un compagnon de chasse a été blessé pendant l'expédition.

Les chants d'annonce possèdent un modèle fixe et sont invariablement chantés en falsetto afin que les paroles puissent être comprises sur une grande distance. Ils sont généralement transmis de père en fils.

Enfin, les Kaiapó emploient le terme de "chant ancien» (me tum ngre-re) pour se référer globalement à tout chant traditionnel. Dans son acception la plus stricte, ce terme désigne une série de chants qui excèdent rarement quelques lignes et dont on dit qu'ils remontent aux temps immémoriaux. Le langage employé dans ces chants est une forme archaïque du kaiapó.

Les chants anciens sont incorporés aux mythes ou aux récits relatant un événement historique. Ils ne sont en fait jamais transmis isolément mais font partie intégrante d'un récit particulier. Je ne connais aucun chant ancien transmis indépendamment d'un récit, c'est-à-dire en dehors du contexte qui lui est propre.

La transmission des mythes et des récits se fait soit au sein des maisonnées, soit dans la maison des hommes. La connaissance des mythes et récits est, en tant que telle, accessible à tout Kaiapó (homme ou femme), mais la capacité de les narrer en public dépend d'une série de facteurs, dont l'éloquence du narrateur et l'intérêt qu'il aurait manifesté envers la tradition orale quand il était encore jeune.

Le chant ancien suivant est invariablement incorporé au mythe relatant comment un ancêtre est allé vivre parmi les poissons où il a assisté à une cérémonie d'imposition de noms. A son retour dans le monde des humains, il transmit à ses congénères les chants et les noms cérémoniels qu'il avait appris parmi les poissons (dans ce cas particulier, tous les noms cérémoniels sont munis du préfixe bep ou bekwỳnh): 
$\hat{O}, \hat{o}, \hat{o}, \hat{o}, \hat{o}$,

nhymmy nhĩ ngônh,

kãm my nã Bep-pe mã bô wajêt-te,

$\hat{o}, \hat{o}, \hat{o}, \hat{o}, \hat{o}$,

tekrwỳdy-re nhĩnhĩ bê Bepryti-re,

ô, ô, êrur, êrur.

$\hat{O}, \hat{o}, \hat{o}, \hat{o}, \hat{o}$,

nhymmy nhĩ ngônh,

kãm my nã Bekwỳnh mã bô wajêt-te,

$\hat{o}, \hat{o}, \hat{o}, \hat{o}, \hat{o}$,

korã-re nhĩnhĩ bê Bekwỳnhkôrã,

$\hat{o}$, ô, êrur, êrur.

Ô, ô, ô, ô, ô,

un homme était dans l'eau,

il a vu une feuille Bep-pe, et la feuille flottait à la surface,

ô, ô, ô, ô, ô,

le nom du piranha est Bepryti-re,

ô, ô, cours, cours.

Ô, ô, ô, ô, ô,

un homme était dans l'eau,

il a vu une feuille Bekwỳnh, et la feuille flottait à la surface,

ô, ô, ô, ô, ô,

le nom du poisson surubi est Bekwỳnhkôrã,

ô, ô, cours, cours.

Les types de chants kaiapó brièvement illustrés dans ces pages correspondent à des circonstances particulières d'interprétation en public et se caractérisent par des modes spécifiques de transmission.

Il est apparu qu'une étude exhaustive de la musique kaiapó fait encore défaut. Que cette contribution ethnographique stimule de futures recherches sur les expressions musicales de cette ethnie dont la survie culturelle est menacée aujourd'hui plus que jamais ! 


\section{Notes}

* Traduit de l'anglais par Isabelle Schulte-Tenckhoff.

1 A ce jour, la musique de deux groupes Gê seulement a fait l'objet d'analyses détaillées, qui sont les Suyâ (Seeger 1979, 1980, 1981 et 1986) et les Xavante (Aytai 1976).

2 Pendant cette période, j'ai passé plus de 33 mois chez les Kaiapó. Ces enquêtes sur le terrain ont été financées par trois institutions belges: la Fondation Léopold III, la Fondation nationale de la recherche scientifique et la Fondation de la vocation. Je tiens à remercier ces institutions de l'assistance dont elles m'ont fait bénéficier, de même que la Fondation national de l'Indien (FUNAI) du Brésil et la Fondation nationale brésilienne pour la recherche $(\mathrm{CNPq})$ pour m'avoir autorisé à me rendre en territoire kaiapó. Je voudrais également remercier M. Laurent Aubert pour avoir commenté des versions antérieures de cet article.

3 Pour l'ethnographie des Kaiapó, voir Dreyfus (1963), Turner (1965), Vidal (1977) et Verswijver (1985).

4 Voir en particulier Seeger $(1976: 353-55 ; 1981)$ et Verswijver $(1985: 84)$.

5 A l'exception des rites funéraires, aucun rite de passage ne s'accompagne de chants ou de danses.

"Hormis la cérémonie d'imposition de noms dite me ni bi'ôk ("femmes peintes") - où les femmes dansent en groupe - les protagonistes sont toujours des hommes (qu'ils soient accompagnés ou non de quelques femmes). Voir Verswijver (1982) pour une description de la cérémonie des «femmes peintes».

7 Voir Verswijver (1983b) pour une analyse détaillée de la complexité des ornements kaiapó.

8 Les Kaiapó utilisent les instruments de musique suivants: des cornes, des flutes, des sifflets, des ceintures ou bâtons munis de sonnailles, des bâtons de percussion et des hochets. L'emploi de tous ces instruments est réservé à des occasions rituelles particulières, à l'exception de la flûte. Cette dernière, dont on joue pour le simple plaisir, a été introduite parmi plusieurs groupes kaiapó à travers des contacts avec les Jurúna, une ethnie voisine.

9 Invariablement, les noms et les privilèges rituels se transmettent de la catégorie parentale ingêt (père de la mère, père du père, frère de la mère) aux tàbdjwỳ hommes (fils du fils, fils de la fille, fils de la sœur) ainsi que de la catégorie kwatyi (mère de la mère, mère du père, sœur du père) aux tàbdjwỳ femmes (fille du fils, fille de la fille, fille du frère). Voir Vidal (1977: 107-112) et Verswijver (1983a) pour une analyse plus détaillée des pratiques kaiapó d'imposition de noms.

${ }^{10}$ Aux yeux des Kaiapó, la sphère du hochet symbolise l'univers.

${ }^{11}$ Cette phrase se réfère aux individus qui ne participent pas souvent aux activités communautaires, mais se bornent à agir dans le cadre de la sphère familiale.

${ }^{12}$ Le terme kaiapó pour "esprit» est karõ, terme également employé pour désigner une ombre ou une image trompeuse.

${ }^{13}$ Occasionnellement, certains vers de tels chants sont interprétés lorsqu'un homme quitte le village et pénètre dans la forêt, ou qu'il se déplace en forêt. A ces moments, les chants sont toujours exécutés en falsetto aigu pour «faire plaisir aux esprits» (karõ kĩnh kadjy).

${ }^{14}$ Voir Verswijver (1981) pour une description détaillée de la cérémonie d'imposition de noms tàkàk/nhàk.

${ }^{15}$ Dans le cas d'une épidémie ou de conflits internes, il arrive que l'une ou l'autre cérémonie majeure soit organisée à des intervalles pouvant atteindre cinq à quinze ans !

${ }^{16}$ Malheureusement je n'ai pas pu vérifier cet aspect important des chants cérémoniels. 


\title{
Bibliographie
}

\author{
AYTAI Desidério \\ 1976 O mundo sonóro Xavante. Thèse de maîtrise. Universidade Católica de Campinas \\ (Brésil).
}

\section{DREYFUS Simone}

1963 Les Kayapó du Nord: contribution à l'étude des Indiens Gê. Paris/La Haye : Mouton.

\section{SEEGER Anthony}

1979 "What can we learn when they sing ? Vocal genres of the Suyá Indians of central Brazil ». Ethnomusicology 23 : 373-394.

1980 «Sing for your sister : the structure and performance of Suyá akia». In: The ethnography of musical performance (McLeod \& Herndon eds). Philadelphia : Norwood, pp. 7-43.

1981 Nature and society in central Brazil : the Suya Indians of Mato Grosso. Cambridge, Mass. : Harvard University Press.

1986 «Oratory is spoken, myth is told, and song is sung, but they are all music to my ears". In: Native South American Discourse (Sherzer \& Urban eds.). Berlin/New York/Amsterdam : Mouton de Gruyter, pp. 59-82.

\section{TURNER Terence}

1966 The social structure and political organization among the Northern Cayapó. Unpublished PhD dissertation, Harvard University (Department of Public Relations).

\section{VERSWIJVER Gustaaf}

1981 «Les gens aux bracelets noirs : un rite de passage chez les Indiens Kayapó du Brésil central ». Naître, vivre et mourir: actualité de Van Gennep (catalogue, Musée d'ethnographie de Neuchâtel), pp. 95-118.

1982 «Les femmes peintes : une cérémonie d'imposition de noms chez les Kaiapó-Mẽkrãgnoti du Brésil central ». Bulletin de la Société suisse des Américanistes (Genève) 46 : 41-59.

1983a «Cycles in Kaiapó Naming Practices». Communication and Cognition (Gand) 16(3): 301-323.

1983b "Essai sur l'usage de la parure chez les Indiens Kaiapó du Brésil central». Bulletin du Musée d'ethnographie (Genève) 25/26:23-62.

1985 Considerations on Mekrãgnoti Warfare. Thèse de doctorat inédite, Université de Gand (Département des sciences sociales).

VIDAL Lux

1977 Morte e vida de uma sociedade indígena brasileira: os Kayapó-Xikrin do Rio Cateté. São Paulo: Editora Hucitec. 